\title{
Microencapsulation of Herb for Food Application
}

\author{
Sawale Pravin Digambar ${ }^{1 *}$, Shendurse $\mathrm{AM}^{2}$ and Changade SP${ }^{1}$ \\ ${ }^{1}$ College of Dairy Technology, Maharashtra Animal and Fishery Science University, India \\ ${ }^{2}$ College of Dairy Science and Food Technology, India
}

*Corresponding author: Pravin Digambar Sawale, 1College of Dairy Technology, College of Dairy Technology, Warud (Pusad)-445204, Nagpur

Submission: 眥 November 03, 2017; Published: 眥January 18, 2018

Keywords: Nutraceutical; Hypocholesterolmic; Anti-carcinogen; Coacervation

\section{Mini Review}

Nutraceutical can be defined as any substance that is consumed as food or a part of a food that provides medical or health benefits including prevention and treatment of disease [1]. Herbal nutraceuticals are commonly used by people who seek alternative health care. The goal of achieving an optimal or maximal state of nutrition and health is becoming an increasing challenge with the introduction of newer categories of nutraceuticals. In recent past, there is rapid upsurge in usage and demand of herbal medicines and food preparation. According to WHO, the estimated world production was 62 billion US dollar which is projected to grow to US $\$ 5$ trillion by 2050 [2].

Research interests have been focused on various herbs e.g. ashavganda, Satavari, vidarikand, arjuna etc. These herbs are very important medicinal plants that have been used in Ayurvedic and indigenous medicine for long time ago. Herbs are chemically rich with its varied content of active compounds such as tannin, phytosterols, saponin, flavonoids (arjunone, arjunolone, luteolin), gallic acid and many useful polyphenols. These herbs can be used as an antioxidant, antithrombotic, hypolipidemic, immunomodulatory activities, liver tonic, diuretic, hypocholesterolmic, anti-carcinogen, anti-inflammatory agent and has been used for centuries to treat a wide range of diseases [3-5]. Ayurveda, Indian traditional medical science found several ways in which the medicinal benefits of herbs could be conveyed via certain foods as carriers. Milk is one of the most important carriers of herb which has been effectively used to deliver phytochemicals for targeted health benefits in the traditional Indian system of medical science [6]. It is therefore envisaged to study the possibility of incorporating Herb extract into milk for developed functional drinks preparation. However, Herb extracts posses an unpleasant flavour, astringent taste and abnormal brown colour characteristics. Microencapsulation of the herb extracts appears to be an alternative for mask flavour, colour and astringency of the extract [7].

Microencapsulation can be prepared by using certain food grade core materials like maltodextrin, WPC, B-cyclodextrin is useful for delivery of bioactive herbal compound [8]. It has two distinct phases; an inner bioactive herbal compound phase, which is encapsulated by second core material phase, has been successfully tried for delivery of certain flavours or active ingredients. The matrix for encapsulation is constructed using food grade or GRAS materials that can fulfill these requirements may include polysaccharides of plant or microbial origin, food proteins, emulsifiers and so on. Emulsion based delivery system also exhibit controlled release behaviour and better bioavailability when ingested as part of food [9].

In food industry, the various microencapsulation techniques solvent dispersion/evaporation, phase separation (coacervation), drying, co-crystallization, and interfacial polymerization have been widely used to protect food ingredients against deterioration, volatile losses or premature interaction with other ingredients [10]. Several materials have been encapsulated in the food industry, including, amino acids, vitamins, minerals, antioxidants, colorants, enzymes and sweeteners [11]. The protective mechanism is to form a membrane (wall system) to enclose droplets or particles of the encapsulated material (core). So far, various kinds of microencapsulation techniques, such as solvent dispersion/ evaporation, phase separation (coacervation), drying cocrystallization, and interfacial polymerization, have been developed [12].

\section{Nanotechnology/Nanoemulsion}

Nanotechnology is an enable technology that has the potential to revolutionize agriculture and food systems. Driven by increasing consumer demand for healthy food products, researchers have been applying tools and knowledge in nanotechnology to address the issues relevant to food and nutrition. Nanoemulsions are a class of extremely small droplets that appear to be transparent or translucent with a bluish coloration. They are usually in the range 50 to $200 \mathrm{~nm}$ but much smaller than the range (from 1 to $100 \mu \mathrm{m}$ ) for conventional emulsions. Nano scale control over food molecules may lead to the modification of many macro scale characteristics, 
such as texture, taste, other sensory attributes, processability, and stability during shelf life. The applications of nanotechnology in food and agricultural systems are growing very fast. One important application of nanotechnology in food and nutrition is to design and development of novel functional food ingredients with improved water solubility, thermal stability, oral bioavailability, sensory attributes, and physiological performance. The nano sizes of emulsions enhance not only stability of the emulsions, but also the bioavailability of the encapsulated phytochemicals.

Nanoemulsions can be prepared through either high or low energy emulsifications. High-energy emulsification methods include high shear homogenization, high-pressure homogenization, microfluidization, ultrasonic homogenization [13], and electrified coaxial liquid jets [14]. It should be pointed out that, although ultrasonic homogenization and electrified coaxial liquid jets could also be used to form nanoemulsions, they are currently limited in laboratory use and have not been used in large batch production $[13,15]$.

High-energy methods are effective in reducing droplet sizes, but may not be suitable for some unstable molecules, such as proteins or peptides. Alternatively, low-energy emulsification methods, such as phase inversion (PIT) method, which uses the changes in solubility of polyoxyethylene-type non-ionic surfactants with temperature $[16,17]$, colloidosomes [18], cubosomes [19], and microfluidic channels [20], can also be used to prepare nanoemulsions. From a food technology perspective, the microencapsulation in wall/ coating polymer or the embedding in polymeric matrix reduces the moisture/water content and acts as a physical barrier to oxygen and small molecules inhibiting chemical and enzymatic degradations [21]. Moreover, microencapsulation, depending on the polymers used, may increase the solubility/dissolution rate of the product and control organoleptic modifications [8].

Common core materials include carbohydrates, gums, semisynthetic cellulose derivatives and synthetic polymers [8,22]). Each carrier has advantages and disadvantages in terms of properties, cost and encapsulation efficiency. Currently, maltodextrins, soluble modified starch derivatives are used alone or in combination with other materials in food and drug processing of plant extracts, aromatic additives, carotenoids and vitamins [23,24]. Maltodextrins have multifaceted functions including bulking and film formation properties, binding ability of flavour and fat, reduction of oxygen permeability of wall matrix. However, its low glass transition temperature, leading crystals formation under increasing temperature [24,25] may induce disruption of the structural integrity of wall matrix and produce agglomeration or caking of micro particulate powders. As a result, release of encapsulated actives and degradation/oxidation process may occur during the storage period. Therefore, a more efficient and stable carrier for natural products.

\section{Conclusion}

During the past decade, the interest in bioactive components including polyphenols, isoflavonoids etc has been increased considerably because of beneficial effects proposed by epidemiologists, nutritionists and food manufacturers. However, these bioactive components is posses unacceptable sensorily properties, light and oxygen sensitive hence incorporation into milk system often leads to a undesirable changes into product. During storage, these changes are pronounced which deteriorate products more which limits its application.

Encapsulation of the herbs appears to be an excellent tool for masking flavour, colour and astringency of the extract. It could enough to hold the bioactive compounds herb extracts which has not only minimizing its undesirable effect but also maintained stability

\section{References}

1. Brower V (1998) Nutraceuticals: poised for a healthy slice of the healthcare market? Nature Biotechnology 16(8): 728-731.

2. Singh H. (2006) Prospects and challenges for harnessing opportunities in medicinal plants sector in India). Law, Environment and Development Journal, pp. 196.

3. Sawale PD, Patil GR, Singh RRB, Arvind K, Ghule AK (2012) Potential Application of Milk and Milk Products as Carrier for Herbs and Nutraceuticals. Current Topics in Nutraceutical Research 10(2): 103111.

4. Sawale PD, Ramesh P, Shaik HA, Anuj K, Kapila S, et al. (2015) Hypolipidemic and Anti-Oxidative Potential of Encapsulated Herb (Terminalia arjuna) Added Vanilla Chocolate Milk in High Cholesterol Fed Rats. J Sci Food Agric 96(4): 1380-1385.

5. Sawale PD, Patil GR, Hussain SA, Singh AK, Singh RRB (2017a) Release Characteristics of Polyphenols from Microencapsulated Terminalia Arjuna Extract: Effects of Simulated Gastric Fluid. International journal of food properties 20(12): 3170-3178.

6. Sawale PD, Patil GR, Hussain SA, Singh AK, Singh RRB (2017b) Effect of incorporation of encapsulated and free Arjuna herb on storage stability of chocolate vanilla dairy drink. Food Bioscience 19: 142-148.

7. Lampronti MTH, Khan M, Borgatti NB, Gambari R (2008) Inhibitory effects of Bangladeshi medicinal plant extracts on interactions between transcription factors \& target DNA sequences. Evidence-Based Complementary and Alternative Medicine 5(3): 303-312.

8. Chiou D, Langrish TAG (2007) Development \& characterization of novel nutraceuticals with spray drying technology. Journal of Food Engineering 82: 84-91.

9. McClements DJ, Decker EA, Park Y, Weiss J (2009) Structural Design Principles for Delivery of Bioactive Components in Nutraceuticals and Functional Foods. Critical. Review in Food Science and Nutrition 49(6): 577-606.

10. Shu B, Yu W, Zhao Y, Liu X (2006) Study on Microencapsulation of Lycopene by Spray Drying. Journal of Food Engineering, 76: 664-669.

11. Shahidi F, Naczk M (2005) Phenolic in food and nutraceuticals. Book Reviews in Trends in Food Science \& Technology 16: 171-176.

12. Champagne CP, Fustier P (2007) Microencapsulation for the improved delivery of bioactive compounds into foods. Current Opinion Biotechnology 18(2): 184-190.

13. Solans C, Izquierdo P, Nolla J, Azemar N, Garcia-CKJ (2005) Nanoemulsions. Curr Opin Colloid In 10(3-4): 102-110.

14. Loscertales G, Barrero A, Guerrero I, Cortijo I, Marquez M, et al. (2002) Micro/Nano Encapsulation via Electrified Coaxial Liquid Jets. Science 295(5560): 1665-1698.

15. Mason TG, Wilking JN, Meleson K, Chang CB, Graves SM (2006) Nanoemulsions: formation, structure, and physical properties. J Phys Condes Matter 18(41): 635-666. 
16. Shinoda K, Saito H (1968) The effect of temperature on the phase equilibria and the types of dispersions of the ternary system composed of water, cyclohexane, and nonionic surfactant. J Colloid Interf Sci 26(1): 70-74.

17. Rang MJ, Miller CA (1999) Spontaneous emulsification of oils containing hydrocarbon, nonionic surfactant, and oleyl alcohol. J Colloid Interface Sci 209(1): 179-192.

18.Dinsmore AD, Hsu MF, Nikolaides MG, Manuel M, Bausch AR, et al. (2002) Colloidosomes: self-assembled, selectively permeable capsules composed of colloidal particles. Science 298(5595): 1006-1009.

19.Spicer P (2004) Bicontinuous cubic liquid crystals nanostrucured particles. In: Nalwa $\mathrm{H}$ (Ed.) Encyclopaedia of nanoscience and nanotechnology. Marcel Dekker, New York, USA, pp. 881-892.

20.Xu QY, Nakajima M, Binksm BP (2005) Preparation of particle-stabilized oil-in water emulsions with the microchannel emulsification method. Colloids Surf A 262(1-3): 94-100.
21. Wang Y, Zhaoxin L, Fengxia L, Xiaomei B (2009) Study on microencapsulation of curcumin pigments by spray drying. European Food Research and Technology 229(3): 391-396.

22. Kha TC, Nguyen MH, Roach PD (2010) Effect of spray-drying conditions on the physicochemical and antioxidant properties of the Gac (Momordica cochinchinensis) fruit aril powder. Journal of Food Engineering 98(3): 385-392.

23. Sadeghi A, Shahidi F, Mortazavi SA, Mahalati MN (2008) Evaluation of different parameters effect on maltodextrin production by - amylase Termamyl 2-x. World Appliance Sciences Journal 3(1): 34-39.

24. Bae KE, Lee SJ (2008) Microencapsulation of avocado oil by spray drying using whey protein and maltodextrin. Journal of Microencapsulation 25(8): 549-560.

25. Drusch S (2006) Sugar beet pectin: A novel emulsifying wall component for microencapsulation of lipophilic food ingredients by spray-drying. Food Hydrocolloids 21(7): 1223-1228. 